\title{
Inflation in Supersymmetric Cosmic String Theories
}

\author{
Anne-Christine Davis \& Mahbub Majumdar \\ Department of Applied Mathematics \& Theoretical Physics (DAMTP), University of Cambridge, Silver Street, Cambridge
}

CB3 $9 E W, U K$.

\begin{abstract}
We examine a non-Abelian SUSY $S U(2) \times U(1)$ gauge theory and a SUSY $U(1)$ theory originally used to investigate the microphysics of cosmic strings in supersymmetric theories. We show that both theories automatically include hybrid inflation. In the latter theory we use a $D$ term to break the symmetry. SUSY is broken during inflation and restored afterwards. Cosmic strings are formed at the end of inflation. The temperature anisotropy is calculated and found to vary as $\left(M_{G U T} / M_{P}\right)^{2}$.
\end{abstract}

\section{INTRODUCTION}

In recent years, supersymmetry (SUSY) has become increasingly favoured as the theoretical structure underlying fundamental particle interactions. In this light it is natural to investigate possible cosmological implications of SUSY.

Until recently, inflation and topological defects were thought of as rival theories for the formation of density perturbations in the Universe. However, in supersymmetric theories it is natural for the two to coexist. In this paper we examine recently proposed supersymmetric models which give rise to cosmic strings [1,2]. We show that these theories are automatically hybrid inflation models. Previous work explored the microphysics of the cosmic strings, but did not consider the resulting cosmology. Whilst the cosmology of mixed models with cosmic strings and inflation has previously been explored, the fact that supersymmetric models with cosmic strings naturally inflate is not so well known.

The theories we examine are based on both nonAbelian and Abelian gauge symmetries, with symmetry breaking arising in two distinct ways. The first model is a $S U(2) \times U(1) \rightarrow U(1) \times Z_{2}$ supersymmetric gauge theory with the gauge symmetry breaking implemented with a $F$ term [1]. The second theory is a SUSY $U(1) \rightarrow Z_{2}$ gauge theory with a Fayet-Illiopoulos $D$ term [2]. In both cases it was previously found that there were fermion zero modes and supersymmetry was broken in the string core. The zero modes in the first theory occurred in pairs, whilst there was a single zero mode in the second theory. The effects of soft-SUSY breaking terms were investigated. In the former case: (1) SUSY breaking Higgs mass terms did not destroy the zero modes; (2) SUSY breaking gaugino mass terms destroyed all zero modes which involved gauginos; (3) SUSY violating trilinear terms created Yukawa couplings which destroyed all zero modes. In the second theory, the zero mode survived soft-SUSY breaking terms. This zero mode could move along the string resulting in the string carrying a current. Such current-carrying strings could cause cosmological problems [3].

The models we consider are particularly attractive for several reasons. (1) They incorporate both cosmic strings and hybrid inflation, which has recently been shown to give good agreement with observations [4.5]. (2) The inflaton field is typically much smaller than $M_{P}$, allowing Planck era physics to be ignored. (3) Inflaton physics can be taken to be normal GUT particle physics. Normally the inflaton potential must suddenly "turn off" to allow inflation to end. For field values less than $M_{P}$, such functions are often complicated. In hybrid inflation, the ending of inflation is simply done by one of the other GUT fields. This simplification allows many types of simple potentials to cause and end inflation. (4) By invoking supersymmetry, coupling constants often do not need to be fine-tuned. 8

In this paper we show that the two models we consider do indeed hybrid inflate and lead to acceptable cosmic microwave anisotropies. Supersymmetry is broken during inflation and a radiative correction to the effective potential ensures that inflation ends. SUSY is restored after cosmic strings are formed, though as previously shown [2], it is broken in the string core. Inflation ends before the GUT phase transition commences since the slow roll condition is violated before the phase transition. Similar results have appeared in different contexts [8 12].

We proceed by reviewing the $S U(2) \times U(1) F$-term SUSY gauge theory and the $D$-term U(1) model. Then we compute the radiative correction to the effective potential for both models. The CMB anisotropy is then calculated. Finally we end with our conclusions.

\section{A $S U(2) \times U(1)$ F-TERM SUSY GAUGE THEORY}

The tree-level effective potential for a SUSY invariant theory is

$$
U=\sum_{i}\left|F_{i}\right|^{2}+\frac{1}{2} \sum_{a} D^{a} D_{a}
$$

where the $F$-term (first term) is $F_{i}=\delta W\left[\phi_{i}\right] / \delta \phi_{i}$, and $W\left[\phi_{i}\right]$ is the superpotential with the fermionic terms set to zero. The $D$ term (second term) is $D^{a}=-(\kappa+$ $g \sum_{i j} q_{a} \phi_{i}^{\dagger}\left(T^{a}\right)_{j}^{i} \phi^{j}$. Here $\kappa$ is a Fayet-Illiopoulos constant and is nonzero if and only if the action possesses a $U(1)$ symmetry. $T^{a}$ is the group generator and the sum in (1) 
is over all group generators. $g$ is the coupling constant and $q_{a}$ is a dimensionless charge.

The $S U(2) \times U(1)$ theory we are considering has the superpotential [1]

$$
W=\mu_{1} S_{0}\left(\boldsymbol{\Phi} \cdot \tilde{\boldsymbol{\Phi}}-\eta^{2}\right)+\mu_{2}\left(S \boldsymbol{\Phi}^{T} \Lambda \boldsymbol{\Phi}+\tilde{S} \tilde{\boldsymbol{\Phi}}^{T} \Lambda \tilde{\boldsymbol{\Phi}}\right)
$$

where $\boldsymbol{\Phi}$ and $\tilde{\boldsymbol{\Phi}}$ transform as $S U(2)$ triplets, and $S_{0}, S, \tilde{S}$ are $U(1)$ scalars.

The tree level effective potential is

$$
\begin{aligned}
U= & \mu_{1}^{2}\left|\phi \cdot \tilde{\phi}-\eta^{2}\right|^{2}+\mu_{2}^{2}\left|2 \phi_{1} \phi_{3}-\phi_{2}^{2}\right|^{2}+\mu_{2}^{2}\left|2 \tilde{\phi}_{1} \tilde{\phi}_{3}-\tilde{\phi}_{2}^{2}\right|^{2}+ \\
& \left|\mu_{1} s_{0} \tilde{\phi}+2 \mu_{2} s \Lambda \phi\right|^{2}+\left|\mu_{1} s_{0} \phi+2 \mu_{2} s \Lambda\right|^{2}+ \\
& e^{2}\left|\left(\phi_{1}+\phi_{3}\right) \phi_{2}^{*}-\left(\tilde{\phi}_{1}+\tilde{\phi}_{3}\right) \tilde{\phi}_{2}^{*}\right|^{2}+ \\
& \frac{e^{2}}{2}\left(\left|\phi_{1}\right|^{2}-\left|\phi_{3}\right|^{2}-\left|\tilde{\phi}_{1}\right|^{2}-\left|\tilde{\phi}_{3}\right|^{2}\right)^{2}+ \\
& \frac{e^{2}}{3}\left(|\phi|^{2}-|\tilde{\phi}|^{2}-2|s|^{2}-2|\tilde{s}|^{2}\right)^{2}
\end{aligned}
$$

The minimum of the potential with regard to variations in the $S U(2)$ fields, $\phi, \tilde{\phi}$, leads to the constraint $\phi_{1}=\tilde{\phi}_{1}$ and $\phi_{2}=\tilde{\phi}_{2}=\phi_{3}=\tilde{\phi}_{3}=0$. The potential then becomes

$$
U=\mu_{1}^{2}\left|\phi_{1}^{2}-\eta^{2}\right|^{2}+2 \mu_{1}^{2}\left|s_{0}\right|^{2}\left|\phi_{1}\right|^{2}
$$

This possesses two minima. A global minimum exists at $\phi_{1}=\eta \equiv M_{s_{0}}, s_{0}=0$ where supersymmetry is unbroken. There is also a SUSY violating local minimum at $\phi_{1}=0, s_{0}>\eta \equiv s_{0}^{c r i t}$. This corresponds to the false vacuum. We will assume a chaotic inflation scenario, where $s_{0}$ is initially a random field and $s_{0}>s_{0}^{\text {crit }}$. Thus the $S U(2)$ fields are initially trapped in a false vacuum and the potential initially is

$$
U=\mu_{1}^{2} \eta^{4}
$$

The potential has no slope prompting $s_{0}$ to roll down to its minimum. However, eternal inflation is averted since SUSY is broken and the tree level potential is no longer protected from radiative corrections which cause inflation to end.

\section{A $U(1) D$-TERM GAUGE THEORY}

In this $U(1)$ theory we assume there exists a nonzero Fayet-Illiopoulos constant, $\kappa$. The superpotential is taken to be [2]

$$
W=\alpha \Phi_{0} \Phi_{+} \Phi_{-}
$$

where $\Phi_{0}, \Phi_{+}, \Phi_{-}$have $U(1)$ charges $0,+1,-1$, respectively.

If we redefine $D^{a}=-g\left(\kappa+\sum_{i j} q_{a} \phi_{i}^{\dagger}\left(T^{a}\right)_{j}^{i} \phi^{j}\right)$, as is often done, the tree-level potential is 8, 12,13.

$$
\begin{aligned}
U= & \alpha^{2}\left|\phi_{0}\right|^{2}\left(\left|\phi_{+}\right|^{2}+\left|\phi_{-}\right|^{2}\right)+\alpha^{2}\left|\phi_{+} \phi_{-}\right|^{2} \\
& +\frac{e^{2}}{2}\left(\left|\phi_{+}\right|^{2}-\left|\phi_{-}\right|^{2}+\kappa\right)^{2} .
\end{aligned}
$$

$U$ possesses two minima - a global minimum at $\phi_{0}=$ $\phi_{+}=0, \phi_{-}=\sqrt{\kappa}$ where supersymmetry is unbroken, and a SUSY violating local minimum at $\phi_{+}=\phi_{-}=$ $0, \phi_{0}>(e / \alpha) \sqrt{\kappa}=\phi_{0}^{c r i t}$. As before, we assume a chaotic inflation scenario and that $\phi_{0}>\phi_{0}^{\text {crit }}$. The fields are in the false vacuum and the potential is

$$
U=\frac{e^{2}}{2} \kappa^{2}
$$

As before this potential has no slope. However, a SUSY breaking induced radiative correction will add a slope.

\section{ONE LOOP RADIATIVE CORRECTIONS TO THE $S U(2) \times U(1)$ AND $U(1)$ THEORIES}

\section{A. $S U(2) \times U(1)$ Theory}

We first consider the one loop radiative correction for the $S U(2) \times U(1)$ theory after SUSY breaking.

At one loop, $\phi$ and $\tilde{\phi}$ receive mass corrections. The $s, \tilde{s}, s_{0}$ fields will not acquire masses. To see this, shift the fields from their expectation values

$$
\begin{array}{rrrr}
s & \rightarrow s^{\prime}+s \quad \tilde{s} \rightarrow \tilde{s}^{\prime}+\tilde{s} \quad s_{0} \rightarrow s_{0}^{\prime}+s_{0} \\
\phi_{i} \rightarrow \phi_{i}^{\prime}+\phi_{i} & \tilde{\phi}_{i} \rightarrow \tilde{\phi}_{i}^{\prime}+\tilde{\phi}_{i}
\end{array}
$$

where we take $s, \tilde{s}, s_{0}, \phi_{i}, \tilde{\phi}_{i}$ to be the classical vacuum expectation values. We assume that we are in the false vacuum dominated case. Thus

$$
s=\tilde{s}=\phi_{i}=\tilde{\phi}_{i}=0 \quad s_{0} \neq 0
$$

Upon substituting the shifted fields, and requiring $\phi_{i}^{\prime}=$ $\tilde{\phi}_{i}^{\prime}$ and $s=\tilde{s}$, the $D$-terms drop out. The potential to leading order is

$$
U=\mu_{1}^{2}\left|\vec{\phi}^{\prime 2}-\eta^{2}\right|^{2}+2 \mu_{1}^{2}\left|s_{0}\right|^{2}\left|\vec{\phi}^{\prime}\right|^{2}+\ldots
$$

To find the masses of the fields substitute

$$
\phi_{i}^{\prime}=\frac{a_{i}+i b_{i}}{\sqrt{2}} .
$$

This gives

$$
\begin{aligned}
U= & 2 \cdot \frac{\left(\mu_{1}^{2}\left|s_{0}\right|^{2}-\mu_{1}^{2} \eta^{2}\right)}{2} \sum_{i} a_{i}^{2} \\
& 2 \cdot \frac{\left(\mu_{1}^{2}\left|s_{0}\right|^{2}+\mu_{1}^{2} \eta^{2}\right)}{2} \sum_{j} b_{j}^{2}+\text { non }- \text { mass terms. }
\end{aligned}
$$

Thus the $a_{i}$ acquire masses $\mu_{1}^{2}\left|s_{0}\right|^{2}-\mu_{1}^{2} \eta^{2}$ and the $b_{i}$ acquire masses $\mu_{1}^{2}\left|s_{0}\right|^{2}+\mu_{1}^{2} \eta^{2}$. (There are two sets of $a_{i}$ fields and two sets of $b_{i}$ - hence the factor of 2.) The $\vec{\phi}$ $(\overrightarrow{\tilde{\phi}})$ field splits up into two fields [9, 13, $, 1, \vec{\phi}+\overrightarrow{\phi^{*}},\left(\overrightarrow{\tilde{\phi}}+\overrightarrow{\tilde{\phi}^{*}}\right)$ 
and $i\left(\vec{\phi}-\overrightarrow{\phi^{*}}\right),\left(i\left(\overrightarrow{\tilde{\phi}}-\overrightarrow{\tilde{\phi}^{*}}\right)\right)$ with masses $\mu_{1}\left|s_{0}\right|^{2} \pm \mu_{1}^{2} \eta^{2}$. We now calculate the masses of the fermion partners.

For a general superpotential

$$
W[\Phi]=f_{i} \Phi_{i}+\frac{m_{i j}}{2} \Phi_{i} \Phi_{j}+\frac{\lambda_{i j k}}{3} \Phi_{i} \Phi_{j} \Phi_{k}
$$

the fermionic mass portion of the Lagrangian is

$$
\mathcal{L}_{m}^{F}=-\frac{1}{2}\left(m_{i j}+2 \lambda_{i j k}\left\langle\phi_{k}\right\rangle\right) \psi_{i} \psi_{j}+\text { H.C }
$$

In our case $m_{i j}=0$ and the only non-zero scalar expectation value is $s_{0}$. Thus,

$$
\mathcal{L}_{m}^{F}=-\mu_{1} s_{0} \psi_{i} \tilde{\psi}_{j}+\text { H.C }
$$

and the fermions have mass $\mu_{1} s_{0}$.

The 1-loop potential is found using the formula 14

$$
U\left(s_{0}\right)_{1-\text { loop }}=\sum \frac{(-)^{F}}{64 \pi^{2}} M_{i}\left(s_{0}\right)^{4} \ln \frac{M_{i}\left(s_{0}\right)^{2}}{\Lambda^{2}}
$$

where $\Lambda$ is an ultraviolet cutoff, and $F= \pm 1$ for bosons/fermions. Inserting the masses and adding in the zeroth order part we have [9]8]

$$
\begin{aligned}
U_{e f f}\left(s_{0}\right)= & \mu_{1}^{2} \eta^{4}\left(1+2 \cdot \frac{\mu_{1}^{2}}{64 \pi^{2}}\left(2 \ln \left(\frac{\mu_{1}\left|s_{0}\right|}{\Lambda}\right)^{2}\right.\right. \\
& \left.+\left(\frac{s_{0}^{2}}{\eta^{2}}-1\right)^{2} \ln \left(1-\frac{\eta^{2}}{s_{0}^{2}}\right)+\left(\frac{s_{0}^{2}}{\eta^{2}}+1\right)^{2} \ln \left(1+\frac{\eta^{2}}{s_{0}^{2}}\right)\right)
\end{aligned}
$$

In the chaotic inflation limit, $s_{0} \gg s_{0}^{\text {crit }}=\eta$, one finds

$$
U_{e f f}\left(s_{0}\right)=\mu_{1}^{2} \eta^{4}\left(1+\frac{\mu_{1}^{2}}{16 \pi^{2}}\left(\ln \left(\frac{\mu_{1}\left|s_{0}\right|}{\Lambda}\right)^{2}+\frac{3}{2}\right)\right) \text {. }
$$

\section{B. U(1) Theory with Fayet-Illiopoulos $D$-term}

The one loop correction for the $U(1)$ theory with a nonzero Fayet-Illiopoulos constant, $\kappa$, is computed just as for the $S U(2) \times U(1)$ theory. As before, the $\phi_{+}$and $\phi_{-}$split up into scalars with masses $\alpha^{2}\left|\phi_{0}\right|^{2} \pm e^{2} \kappa$. The one loop potential in the limit of a large inflaton field $\phi_{0}$ is 13,8

$$
U_{e f f}\left(\phi_{0}\right)=\frac{e^{2} \kappa^{2}}{2}\left(1+\frac{e^{2}}{16 \pi^{2}}\left(\ln \left(\frac{\alpha\left|\phi_{0}\right|}{\Lambda}\right)^{2}+\frac{3}{2}\right)\right) .
$$

\section{THE END OF INFLATION}

Inflation occurs as long as the slow-roll parameters $\epsilon, \eta$ remain small, $(\epsilon, \eta<<1)$, where [7]

$$
\epsilon=\frac{M_{P}^{2}}{16 \pi}\left(\frac{U_{e f f}^{\prime}}{U_{e f f}}\right)^{2} \quad \eta=\frac{M_{P}^{2}}{8 \pi} \frac{U_{e f f}^{\prime \prime}}{U_{e f f}}
$$

The same conclusion holds for the $U(1)$ theory with a

Writing $s_{0}=x s_{0}^{c r i t}$ and using

$$
U_{e f f}^{\prime}=\frac{\mu_{1}^{4} M_{s_{0}}^{3}}{32 \pi^{2}} 4 x\left(\left(x^{2}-1\right) \ln \left(1-\frac{1}{x^{2}}\right)+\left(x^{2}+1\right) \ln \left(1+\frac{1}{x^{2}}\right)\right)
$$

In the limit of large $x$, this is

$$
U_{\text {eff }}^{\prime} \approx \frac{\mu_{1}^{4} M_{s_{0}}^{3}}{8 \pi^{2}} \frac{1}{x} .
$$

We find for the $S U(2) \times U(1)$ case 9

$$
\begin{aligned}
& \epsilon=\left(\frac{\mu_{1}^{2} M_{P}}{8 \pi^{2} M_{s_{0}}}\right)^{2} \frac{x^{2}}{16 \pi}\left(\left(x^{2}-1\right) \ln \left(1-\frac{1}{x^{2}}\right)+\left(x^{2}+1\right) \ln \left(1+\frac{1}{x^{2}}\right)\right)^{2} \\
& \eta=\left(\frac{\mu_{1} M_{P}}{4 \pi M_{s_{0}}}\right)^{2} \frac{1}{4 \pi}\left(\left(3 x^{2}-1\right) \ln \left(1-\frac{1}{x^{2}}\right)+\left(3 x^{2}+1\right) \ln \left(1+\frac{1}{x^{2}}\right)\right)
\end{aligned}
$$

where we approximated $U_{\text {eff }}=\mu_{1}^{2} \eta^{4}$. In the limit of large $x$

$$
\begin{aligned}
\epsilon & =\frac{\mu_{1}^{4}}{1024 \pi^{5}}\left(\frac{M_{P}}{M_{s_{0}}}\right)^{2} \frac{1}{x^{2}} \\
\eta & =-\frac{\mu_{1}^{2}}{64 \pi^{3}}\left(\frac{M_{P}}{M_{s_{0}}}\right)^{2} \frac{1}{x^{2}} .
\end{aligned}
$$

For $x \gg 1$, both $\eta, \epsilon$ are small. For $x \approx 1$, which corresponds to the GUT phase transition, both $\epsilon, \eta$ diverge. Hence inflation ends 90 before the phase transiFayet-Illiopoulos $D$-term.

\section{PREDICTED CMB ANISOTROPIES OF THE $S U(2) \times U(1)$ AND $U(1)$ THEORIES}

\section{A. $S U(2) \times U(1)$ theory}

The temperature quadrapole anisotropy due to inflation can be found to be 15

$$
\begin{aligned}
\left(\frac{\Delta T}{T}\right)_{i n f l} & \left.\approx \sqrt{\frac{32 \pi}{45 M_{P}^{6}}} \frac{U_{\text {eff }}^{3 / 2}}{U_{\text {eff }}^{\prime}}\right|_{x_{Q}} \\
& \approx \sqrt{\frac{32 \pi}{45} \frac{8 \pi^{2}}{\mu_{1}}\left(\frac{M_{s_{0}}}{M_{P}}\right)^{3} x_{Q}}
\end{aligned}
$$

where we have used equation (21). This can be written in terms of, $N_{Q}$, the number of e-folds before the end of inflation at which the Hubble radius crossed the de-Sitter horizon. We have

$$
\begin{aligned}
N_{Q} & =-\frac{8 \pi}{M_{P}^{2}} \int_{Q}^{e n d} \frac{U\left(s_{0}\right)}{U^{\prime}\left(s_{0}\right)} d s_{0} \\
& \approx \frac{32 \pi^{3}}{\mu_{1}^{2}}\left(\frac{M_{s_{0}}}{M_{P}}\right)^{2}\left(x_{Q}^{2}-x_{e n d}^{2}\right) \\
& \approx \frac{32 \pi^{3}}{\mu_{1}^{2}}\left(\frac{M_{s_{0}}}{M_{P}}\right)^{2} x_{Q}^{2}
\end{aligned}
$$


where we have used the approximation equation (21).

Hence

$$
\left(\frac{\Delta T}{T}\right)_{i n f l} \approx \sqrt{\frac{8 \pi}{45}} \sqrt{8 \pi N_{Q}}\left(\frac{M_{s_{0}}}{M_{P}}\right)^{2}
$$

The $\mathrm{SU}(2)$ phase transition produces cosmic strings which contribute to the CMB anisotropy. The anisotropy due to strings can be approximated as 16

$$
\left(\frac{\Delta T}{T}\right)_{\text {string }} \approx 9 G \mu
$$

where $\mu$ is the string mass per unit length. We will approximate

$$
\mu \approx M_{s_{0}}^{2}
$$

The combined temperature anisotropy is then [11

$$
\begin{aligned}
\left(\frac{\Delta T}{T}\right)_{t o t} & \approx \sqrt{\left(\frac{\Delta T}{T}\right)_{\text {infl }}^{2}+\left(\frac{\Delta T}{T}\right)_{\text {string }}^{2}} \\
& \approx \sqrt{81+\frac{8 \pi}{45} \cdot 8 \pi N_{Q}}\left(\frac{M_{s_{0}}}{M_{P}}\right)^{2}
\end{aligned}
$$

We will assume that $N_{Q} \approx 60$. From the CMB temperature anisotropy value of $10^{-5}$ we find $M_{s_{0}} \approx 7 \cdot 10^{15} \mathrm{GeV}$. We can estimate $\mu_{1}$ from equation (24). If the Hubble radius crosses the de-Sitter horizon at $x_{Q} \approx 10$ we find $\mu_{1} \approx 2 \cdot 10^{-2}$.

The spectral index is [9]

$$
\begin{aligned}
n & =1-6 \epsilon+2 \eta \\
& \approx 1-\frac{1}{N_{Q}}=0.98 .
\end{aligned}
$$

where we have used (22) and the result $\mu_{1} \ll 1$

\section{B. $U(1)$ Theory with Non-Zero Fayet-Illiopoulos Term}

Calculations of the CMB anisotropy for the $U(1)$ Fayet-Illiopoulos case is identical to the $S U(2) \times U(1)$ case except the string tension, $\mu$, is identified as [8]

$$
\mu \approx M_{\phi_{-}}^{2} \approx 2 \pi \kappa
$$

since the phase transition occurs at $\left\langle\phi_{-}\right\rangle=\sqrt{\kappa}=M_{\phi_{-}}$.

The CMB anisotropy then constrains $\kappa \approx 8 \cdot 10^{30}$ $(\mathrm{GeV})^{2}$.

\section{COMMENTS AND CONCLUSIONS}

We have examined two SUSY models which lead to strings and found that that the models inflate - via hybrid inflation. The two models show the different ways SUSY breaking can produce and end inflation - by a nonzero vev of a $F$ term, or a $D$ term. A $F$-term Abelian theory would produce very similar results - hence we have not included it in the analysis. We believe hybrid inflation to be a somewhat generic feature of SUSY unified theories. In fact, Jeannerot claims that hybrid inflation is common to most unified theories which exclude gravity with interactions of rank greater than or equal to five [12]. Our results together with [11] generalise this and show that supersymmetric theories with cosmic strings in general, are hybrid inflation models.

The CMB anisotropy of our models was found to be of order $\left(M_{s_{0}} / M_{P}\right)^{2}$. This is of the right magnitude, and allows GUT scale physics to explain the CMB.

However, it is not clear how to extract the same inflation from a higher energy theory such as supergravity or superstring theory. $F$ term models in supergravity tend to cause all scalar fields to be renormalised, even the inflaton. This causes problems. It may destabilise the flat directions and cause the slow-roll conditions to be violated [13]. $D$ term supergravity models do not suffer from this flaw. But any theory relying on $D$ term inflation must not be semi-simple. It's gauge group must contain a $U(1)$ factor. Further, to preserve the flat directions of global SUSY, all fields charged by the Fayet-Illiopoulos $U(1)$ must be driven to zero. This is not always possible. Also, in the context of superstring theory, if the $D$-term dominates any $F$-term during inflation a runaway dilaton problem may occur. [8]

Nevertheless, we believe that SUSY hybrid inflation is sufficiently attractive to warrant further investigation. We consider it worthwhile to deduce whether hybrid SUSY inflation can easily fit into a unified theory including gravity.

One caveat to our results is that the cosmic strings in the $D$-term theory contain fermion zero modes that survive supersymmetry breaking. Such strings could cause potential cosmological problems with the over production of vortons [3]. Also, in the usual cosmic string scenario, the Abelian Higgs model is used as a prototype theory. Recently this has been used in mixed string and inflation scenarios [4,5]. However, the evolution of cosmic strings in realistic theories, such as those considered in this paper, is an open question and deserves investigation.

\section{ACKNOWLEDGEMENTS}

This work was supported in part by PPARC. M.M. is grateful to the Cambridge Commonwealth Trust, Hughes Hall, and DAMTP for financial support while this work was being completed. 
[1] S.C. Davis, A.C. Davis, M. Trodden, Cosmic Strings, Zero Modes and SUSY breaking in Non-Abelian $\mathrm{N}=1$ Gauge Theories, Phys. Rev. D57, 5184 (1998).

[2] S. Davis, A. C. Davis, M. Trodden, N=1 Supersymmetric Cosmic Strings, Phys. Lett. B405, 257 (1997).

[3] R. Bradenberger, B. Carter, A. C. Davis, M. Trodden, Cosmic Vortons and Particle Physics Constraints, Phys. Rev. D54 6059 (1996).

[4] C. Contaldi, M. Hindmarsh, J. Magueijo, CMB and density fluctuations from strings plus inflation, Phys. Rev. Lett. 822034 (1999).

[5] R. A. Battye, J. Weller, Cosmic Structure in Hybrid Inflation Models, astro-ph/9810203.

[6] A. Linde, Hybrid Inflation, Phys. Rev. D49 748 (1994).

[7] E. Copeland, A. Liddle, D. Lyth, E. Stewart and David Wands, Phys. Rev. D49, 6410 (1994).

[8] D. Lyth, A. Riotto, Particle Physics Models of Inflation and the Cosmological Density Perturbation, to appear in Physics Reports, hep-ph/9807278.

[9] G.Dvali, Q. Shafi, R. Shaefer, Large Scale Structure and Supersymmetric Inflation Without Fine Tuning, Phys. Rev. Lett 73, 1886 (1994).

[10] G. Dvali, Inflation Induced SUSY Breaking and Flat Vacuum Direction, Phys. Lett. B355, 78 (1995).

[11] R. Jeannerot, A Supersymmetric SO(10) Model with Inflation and Cosmic Strings, Phys. Rev. D53, 5426 (1996).

[12] R. Jeannerot, Inflation in Supersymmetric Unified Theories, Phys. Rev. D56, 6205 (1997).

[13] P. Binetruy, G. Dvali, D-Term Inflation, Phys. Lett. B388, 241 (1996).

[14] S. Coleman, E. Weinberg, Phys. Rev. D7, 1888 (1973).

[15] A. Liddle, D. Lyth, Physics Reports 231, 1 (1993).

[16] B. Allen et al, Phys. Rev. Lett. 792624 (1997). 